\title{
Study on traditional Chinese medicine medication for acne of wind-heat type in lung meridian based on data mining and network pharmacology
}

\author{
Yitong Yue ${ }^{1}$, Shengping Xue ${ }^{1, *}$, Zhanjun Yue ${ }^{2}$, Weijin Fang ${ }^{1}, \mathrm{Kang}_{\mathrm{Li}}{ }^{1}$, and Jiaqi Gao ${ }^{1}$ \\ ${ }^{1}$ College of Biological Science and Engineering, Hebei University of Economics and Business, Shijiazhuang, China \\ ${ }^{2}$ Yuncheng Zhanjun Hospital of Traditional Chinese Medicine, Yuncheng, China
}

\begin{abstract}
Acne vulgaris of wind-heat in lung meridian is a common skin disease, and there are many traditional Chinese medicine prescriptions, but the mechanism is still not clear. In this paper, the literature on the treatment of acne due to wind-heat in lung meridian and the prescriptions in the patents were collected and sorted out in the past twenty years. Excel 2019 was used for medication frequency statistics, and IBM SPSS 25.0 was used for clustering analysis to obtain the core formula. Network pharmacology was used to collect the related targets of drugs and diseases, to construct the action network, and to conduct enrichment analysis. A total of 137 prescriptions and 167 drugs were obtained. The core prescriptions were Cortex mori, Folium eriobotryae, Scutellariae radix, Glycyrrhrizae radix, Fructus gardeniae, Radix rehmanniae, and Cortex moutan. The treatment of acne due to wind-heat in lung meridian should begin with clearing heat, purging the lung, cooling blood and detoxicating. The core prescriptions mainly played a role through AKT1, IL6, TP53, TNF, VEGFA, EGF targets and Kaposi's sarcoma-related herpes virus infection, measles, toxoplasmosis, EB virus infection, IL-17, MAPK and other signaling pathways, so as to provide reference for further clinical research.
\end{abstract}

\section{Introduction}

Acne is a common chronic skin disease ${ }^{[1]}$, which can be divided into four syndrome types in TCM syndrome differentiation, including wind-heat in lung meridian, damp-heat in gastrointestinal tract, stagnation heat in liver meridian, and phlegm and blood stasis accumulation $^{[2]}$. Wind-heat type of lung meridian is very common and belongs to the early stage of acne, with papules and acne as the main symptoms ${ }^{[3]}$. Traditional Chinese medicine (TCM) culture is extensive and profound. Many famous physicians have their own compatibility of traditional Chinese medicines for acne due to wind-heat in lung meridian. However, due to the complex effect of the traditional Chinese medicine compound, the key components and targets as well as the mechanism for exerting the efficacy in human body are still not clear. In recent years, data mining ${ }^{[4]}$ and network pharmacology technologies have been playing an increasingly important role in the field of traditional Chinese medicine. Therefore, in this study, we explored the medication law of Chinese medicine in the treatment of acne due to wind-heat in lung meridian on the basis of data mining, obtained the core formula, and explored the action mechanism of the core formula from the perspective of network pharmacology, to provide a scientific basis for systematic pharmacological research and clinical medication.

\section{Materials and methods}

\subsection{Sources of literature}

Retrieving the clinical research articles and patents on the treatment of acne due to wind-heat in lung meridian (ACNE) in traditional Chinese medicine (TCM) from January 1, 2000 to September 1, 2020 included in the CNKI China Journal Full-text Database and Wanfang Database and make statistical analysis.

\subsection{The standardization of prescription components of Chinese medicine}

Standardized changes of Chinese medicinal names were made with reference to Pharmacopoeia of the People's Republic of China (2020 edition) ${ }^{[5]}$.

\subsection{Data analysis}

The frequency analysis was performed using Microsoft Excel 2019 to obtain high-frequency drugs, Clustering analysis was performed using IBM SPSS Statistics 25.0.

\footnotetext{
*orresponding author: 774553506@qq.com
} 


\subsection{Prediction of core Chinese medicinal components and targets}

The components of seven Chinese medicinal materials including Radix scutellariae, Radix glycyrrhizae, Mori cortex, Eriobotryae folium, Cortex moutan, Gardeniae fructus, and Dried rehmannia root were searched using TCMSP database (http://tcmspw.com/index.php) ${ }^{[6]}$. The searched Chinese medicinal components were screened by the Oral bioavailability (OB) and the Drug-likeness $(\mathrm{DL})^{[7]}$.

Protein name-gene name conversion was performed using the Uniprot database.

\subsection{Collection of disease targets}

Search the genecards (http://www.genecards.org/) and omim (https://www.omim.org/) databases for "acne." After merging the disease database targets, delete the duplicate values to get the acne vulgaris target.

\subsection{Acquisition of core action targets and protein-protein interaction network diagram analysis}

The common target of drugs and diseases was the potential target. The Chinese medicine target obtained above was matched with the acne target ${ }^{[8]}$, and a proteinprotein interaction (PPI) network model was constructed using STRING 11.0(https://string-db.org) and visualized by Cytoscape 3.7.2 software.

\subsection{Construction and analysis of Chinese medicine-active ingredient-action target network}

The network model of core Chinese medicines, Chinese medicine components and action targets was constructed using Cytoscape 3.7.2 software. Different types of Chinese medicine, components and target nodes were distinguished by selecting different colors and shapes.

\section{$2.8 \mathrm{GO}$ and KEGG analysis}

The Metascape platform (https://david.ncifcrf.gov/) was used for GO (gene ontology) annotation and KEGG (Kyoto Encyclopedia of Gene sand Genomes) enrichment analysis of the core targets, and the $p$ value was set to $<0.01$.

\section{Outcome}

\subsection{Chinese medicine frequency statistics and category analysis}

A total of 292 articles and 10 patents were retrieved. A total of 137 prescriptions were screened out and 167 Chinese medicines were included in the total prescriptions. Four Chinese medicinal herbs with the frequency over $50 \%$ were selected, including Radix scutellariae, Radix glycyrrhizae, Mori cortex, and Eriobotryae folium, as shown in Table 1. These herbs are most commonly used in the combination of clinical prescriptions.

Table 1. Drug frequency, meridian tropism, nature and taste chart with frequency $>40$

\begin{tabular}{lllll}
\hline $\begin{array}{l}\text { Traditional Chinese } \\
\text { medicine }\end{array}$ & $\begin{array}{l}\text { Occurrence } \\
\text { number }\end{array}$ & $\begin{array}{c}\text { frequency } \\
(\%)\end{array}$ & Channels & Sexual flavor \\
\hline $\begin{array}{l}\text { Scutellaria } \\
\text { baicalensis georgi }\end{array}$ & 85 & 62.04 & $\begin{array}{l}\text { Lung, gallbladder, spleen, large } \\
\text { intestine, small intestine }\end{array}$ & Bitter, cold \\
licorice & 81 & 59.12 & Heart, lung, spleen and stomach & Sweet, flat \\
White mulberry & 72 & 52.55 & lung & Sweet, cold \\
Loquat leaf & 69 & 50.36 & Lung, stomach & Bitter, cold \\
St John's wort & 67 & 48.91 & Lung, heart, small intestine & Bitter, cold \\
honeysuckle & 61 & 44.53 & Lung, heart, stomach & Sweet, cold \\
$\begin{array}{l}\text { Raw } \\
\text { dried rehmannia root }\end{array}$ & 52 & 37.96 & Heart, liver, kidney & Sweet, cold \\
Cortex moutan & 50 & 36.50 & Heart, liver, kidney & Bitter, acrid, slightly cold \\
Salvia miltiorrhiza & 49 & 35.77 & Heart, liver & Bitter, slightly cold \\
gardenia & 48 & 35.04 & Heart, lung, tricoke & Bitter, cold \\
Hedyotis diffusa & 47 & 34.31 & Heart, liver and spleen & Bitter, sweet, cold \\
Radix paeoniae rubra & 47 & 34.31 & liver & Bitter, slightly cold \\
The dandelion & 44 & 32.12 & Liver, stomach & Bitter, sweet, cold \\
\hline
\end{tabular}

The total number of occurrences of all Chinese medicinals was 1569, and the largest amounts were found in order of clearing heat and detoxicating, clearing heat and cooling blood, clearing heat and drying dampness, and relieving cough and asthma by category statistics.

\subsection{High frequency drug system cluster analysis}

Get 6 combinations. Combination 1: Mori cortex, Eriobotryae folium, Radix scutellariae, Radix glycyrrhizae, Gardeniae fructus, Dried rehmannia root, 
and Cortex moutan; Combination 2: Rhizoma coptidis, Radix et Rhizoma rhei, and Cortex phellodendri; Combination 3: Herba taraxaci, Herba violae, Flos lonicerae, Prunellae spica, and Fructus forsythiae; Combination 4: Radix paeoniae rubra, Flos chrysanthemi indici and Radix angelicae sinensis; Combination 5: Herba hedyotidis diffusae, Saviae miltiorrhizae radix, Gypsum fibrosum, and Fructus crataegi; Combination 6: Coicis semen and Poria. Through analysis, it was found that the Chinese medicines in Combination 1 were also the most frequently used ones for the treatment of acne due to wind-heat in lung meridian. From the components, they could be regarded as the modified formula of Pipa Qingfei Decoction, indicating that Pipa Qingfei Decoction still occupied the main position in the prescriptions for the treatment of acne due to wind-heat in lung meridian in the past 20 years. Cluster analysis tree diagram as shown in figure 1.

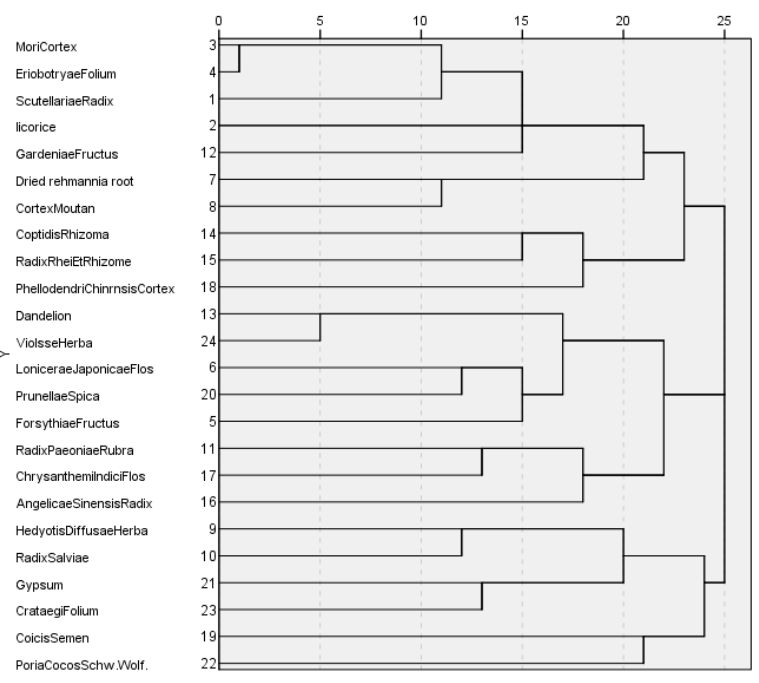

Fig. 1. High-frequency drug clustering analysis diagram

\subsection{Drug component collection and screening of corresponding action targets}

A total of 182 chemical components were obtained through screening with $\mathrm{OB} \geq 30 \%$ and $\mathrm{DL} \geq 0.18$, including 36 from Radix scutellariae, 32 from Mori cortex, 18 from Eriobotryae folium, 1 from Dried rehmannia root, 11 from Cortex moutan, and 15 from Gardeniae fructus. Sixty-nine active components of
Radix et rhizoma glycyrrhizae were screened with $\mathrm{OB} \geq$ $40 \%$ and $\mathrm{DL} \geq 0.18$. OB and DL, components of Dried rehmannia root $\mathrm{B}(\mathrm{OB}=64.62 \%, \mathrm{DL}=0.11)$ and catalpol $(\mathrm{OB}=5.07 \% \%, \mathrm{DL}=0.44)$ were not qualified for screening, but they were also important components of Dried rehmannia root, so they were included in the study. The common components and ADME attributes between Chinese medicinals are shown in Table 2.

Table 2. Information table of partial Chinese medicine components

\begin{tabular}{cllll}
\hline MolID & \multicolumn{1}{c}{ Name } & OB $(\%)$ & DL & \multicolumn{1}{c}{ Component source } \\
\hline MOL000422 & kaempferol & 41.88 & 0.24 & Loquat leaves, mulberry bark, peony bark, gardenia, licorice \\
MOL000098 & quercetin & 46.43 & 0.28 & Loquat leaves, mulberry bark, peony bark, gardenia, licorice \\
MOL000211 & Mairin & 55.38 & 0.78 & Loquat leaves, mulberry bark, peony bark, licorice \\
MOL000358 & beta-sitosterol & 36.91 & 0.75 & Scutellaria, mulberry bark, loquat leaf, gardenia \\
MOL000449 & Stigmasterol & 43.83 & 0.76 & Radix Scutellariae and Gardenia \\
MOL000359 & sitosterol & 36.91 & 0.75 & Scutellariae, peony bark \\
MOL004912 & Glabrone & 52.51 & 0.5 & Mulberry bark, licorice \\
MOL000354 & isorhamnetin & 49.6 & 0.31 & Loquat leaves, licorice \\
\hline
\end{tabular}

After target protein-gene name conversion, the respective targets of the seven Chinese medicines were obtained, as shown in Table 3.

Table 3. Prediction of core drug components and targets

\begin{tabular}{lll}
\hline $\begin{array}{l}\text { Traditional Chinese } \\
\text { medicine }\end{array}$ & $\begin{array}{l}\text { Number of activ } \\
\text { e ingredients }\end{array}$ & $\begin{array}{l}\text { Number of } \\
\text { target }\end{array}$ \\
\hline Loquat leaf & 14 & 273
\end{tabular}

\begin{tabular}{lll} 
licorice & 68 & 227 \\
Gardenia & 12 & 196 \\
White mulberry & 29 & 190 \\
$\begin{array}{l}\text { Cortex moutan } \\
\begin{array}{l}\text { Scutellaria baicalensis } \\
\text { georgi }\end{array}\end{array}$ & 7 & 168 \\
$\begin{array}{l}\text { Raw } \\
\text { dried rehmannia root }\end{array}$ & 3 & 123 \\
\hline
\end{tabular}




\subsection{Acquisition of potential action targets of core traditional Chinese medicines on acne vulgaris}

A total of 1404 targets related to acne vulgaris were obtained, which were matched with the potential targets of seven Chinese medicines, and 122 common targets were obtained. The statistical results of the targets and active components acted on by each Chinese medicine are shown in Table 4.

Table 4. Active components and targets of medicaments for treating acne

\begin{tabular}{ccc}
\hline $\begin{array}{c}\text { Traditional Chinese } \\
\text { medicine }\end{array}$ & $\begin{array}{c}\text { Number of active ingr } \\
\text { edients }\end{array}$ & $\begin{array}{c}\text { Number of } \mathrm{t} \\
\text { arget }\end{array}$ \\
\hline White mulberry & 25 & 126 \\
Loquat leaf & 11 & 106 \\
licorice & 68 & 101 \\
Gardenia & 11 & 83 \\
Cortex moutan & 6 & 82
\end{tabular}

Scutellaria baicalensis

georgi
Raw dried
hmannia root

It could be seen that Mori cortex and Eriobotryae folium had the most corresponding targets, and they played an important role in the prescriptions.

\subsection{Construction and analysis of Chinese medicine-active ingredient-action target network}

The network model of core Chinese medicines, Chinese medicine components and action targets is shown in Fig. 2. A total of 259 nodes and 1805 edges are generated in the graph. The larger the degree value was, the larger the node was and the darker the color was, indicating that more target genes corresponded to the component. It could be seen that MOL000098 quercitrin, MOL000422 kaempferol and MOL000358 $\beta$-sitosterol had the most corresponding gene targets, suggesting that they played an important role in the treatment of diseases.

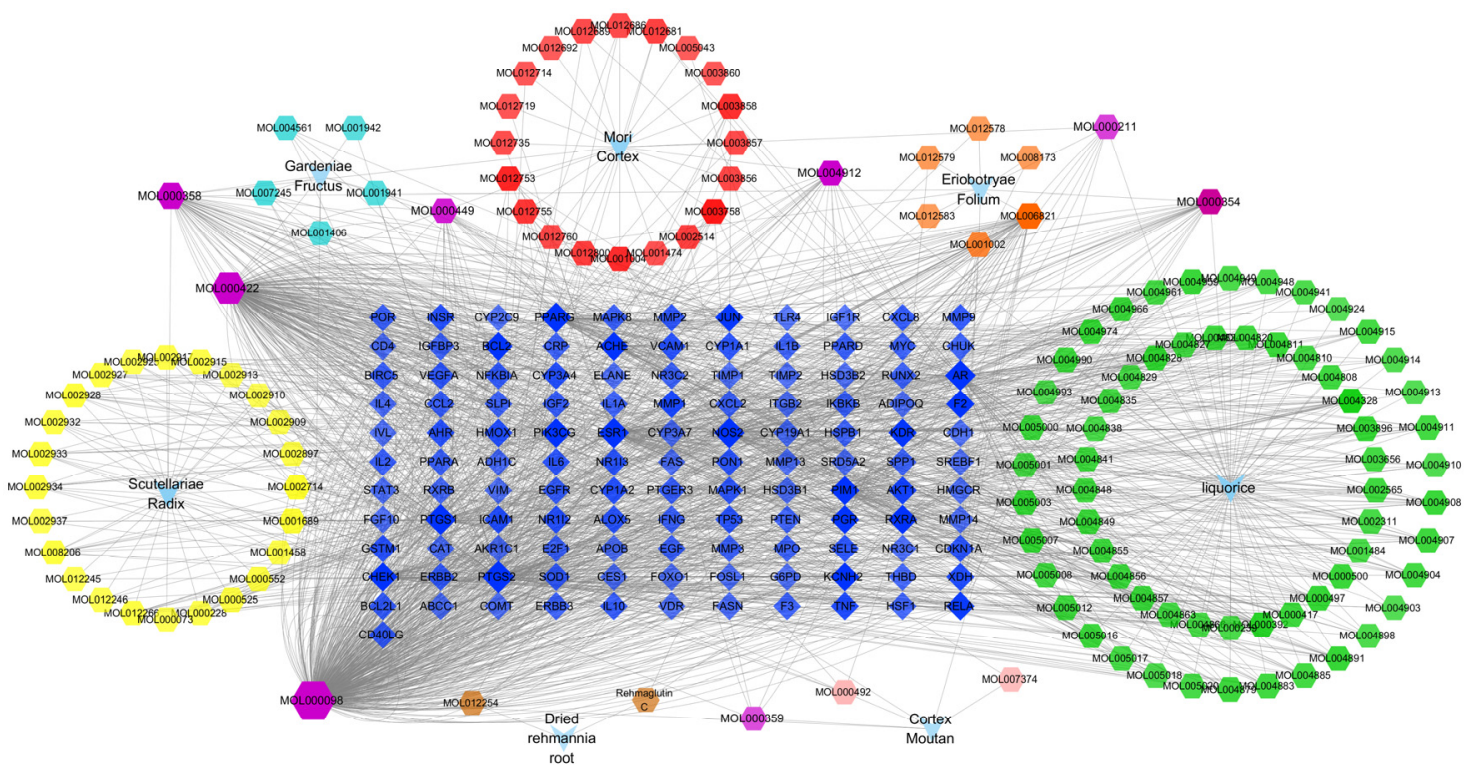

Fig. 2. Chinese medicine-active ingredients-action target network diagram

\subsection{Analysis of protein-protein interaction network of potential targets common to drugs and diseases}

The PPI network constructed by the STRING database is shown in Fig. 3, and contains a total of 122 nodes. The bigger and redder the node, the better the network connectivity.

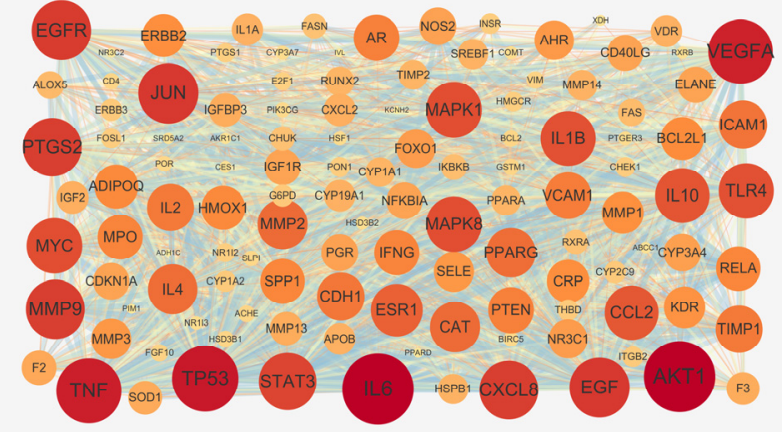

Fig.3. Interaction network diagram of core target protein

After statistical analysis of the topological properties of the nodes, it was found that the key genes AKT1, IL6, 
TP53, TNF, VEGFA, EGF, and JUN played a greater role, and they were core nodes, suggesting that they might play a key role in the treatment of acne vulgaris due to wind-heat in lung meridian.

\subsection{Enrichment analysis of target function and pathway}

A total of 562 entries were obtained from GO functional enrichment analysis, including 362 biological processes, 80 cell compositions, and 120 molecular functions. The pathways with more enrichment targets and lower $\mathrm{P}$ value were more closely related to the treatment of acne vulgaris of wind-heat type in lung meridian with core prescriptions of Chinese medicine.

The results of GO analysis are shown in Fig. 4. The biological processes in which the TCM prescription for acne due to wind-heat in lung meridian mainly participated included cell response to lipids, cell response to organic cyclic compounds, cell response to hormone stimulation, intracellular receptor signaling pathway, and transcription initiation of deoxyribonucleic acid template. The functions of related targets in regulating acne are mainly enriched in nuclear receptor activity, lipidbinding steroid binding, oxidoreductase activity, heme binding, cofactor binding, etc. The cell composition items mainly cover membrane rafts, extracellular matrix, extracellular matrix containing collagen, receptor complex, plasma membrane protein complex, plasma membrane receptor complex, etc.
The KEGG enrichment analysis yielded 280 pathways, of which the bubble diagram of the first 20 pathways was shown in Fig. 5. Where a larger Rich factor value indicates more obvious enrichment. We screened the pathways related to the treatment of acne vulgaris due to wind-heat in lung meridian from the images, including herpes virus infection related to Kaposi's sarcoma, measles, toxoplasmosis, EB virus infection, IL-17, MAPK, NF-kB signaling pathway, etc. Interleukin 17 signal pathway plays an important role in that inflammatory response ${ }^{[9]}$. Through analysis, the core traditional Chinese medicine prescription mainly plays a role by regulating CHUK, CXCL2, IFNG, IKBKB, IL1B, IL4, IL6, CXCL8, JUN, MMP1, MMP3, MMP9, MMP13, NFKBIA, MAPK1, MAPK8, PTGS2, etc. Kaposi's sarcoma-associated herpesvirus produces several signaling molecules during lytic replication, such as vGPCR, vIL6, vIRFs, and K15, that are associated with the inflammatory phenotype observed in KS injury. In the interleukin (IL)-17 producing pathway, Th17 differentiation causes the increase of TGF- $\beta$, interleukin 6 , and interleukin $-1^{[10]}$. The Chinese medicinal prescription acts on this pathway and exerts the effect by regulating AHR, CD4, CHUK, IFNG, IKBKB, IL1B, IL2, IL4, IL6 and MAPK1 genes. NF-kB signaling pathway is related to the regulation of immunity and inflammation. Acne is induced by TNF- $\alpha$, IL-1 or by- products of bacterial and viral infections. The Toll-like receptor signaling pathway is also an important pathway that causes pro-inflammatory cytokine production ${ }^{[11]}$.

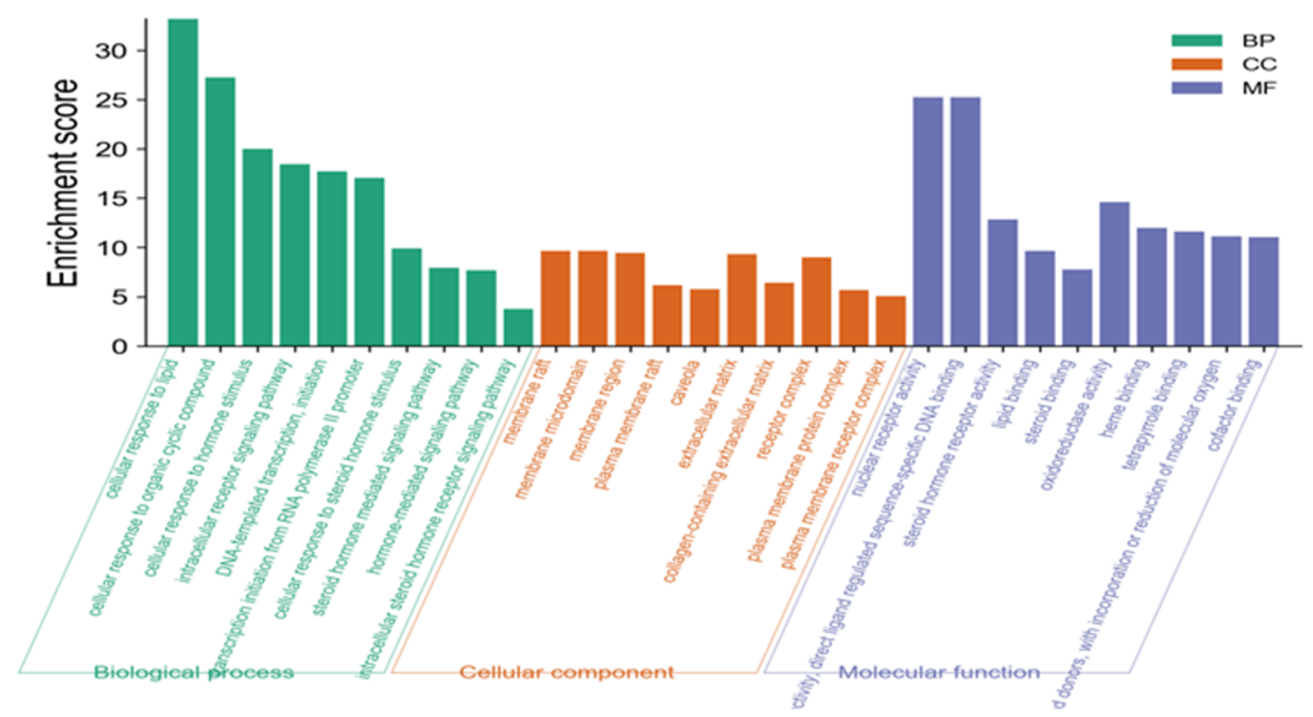

Fig. 4. Histogram of GO triple enrichment analysis

Chinese medicine mainly regulates AKT1, CHUK, IKBKB, IL6 and other genes to control the disease. 


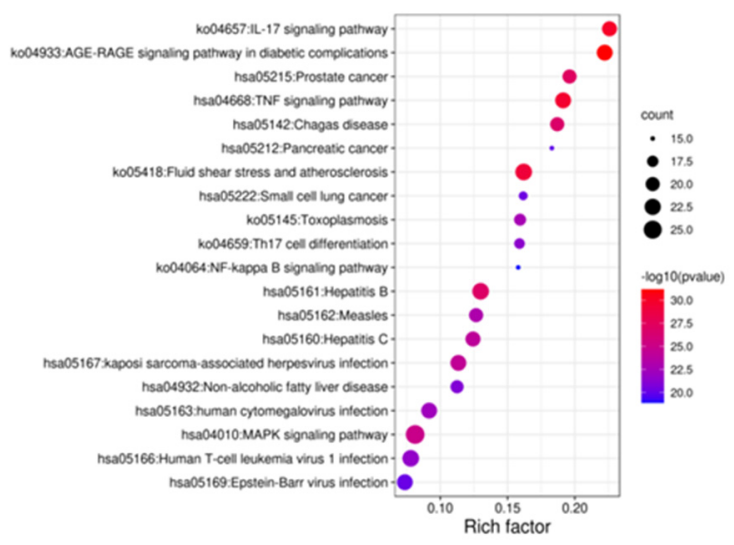

Fig. 5. KEGG enrichment analysis bubble chart

\section{Conclusions}

In this study, data mining was used to analyze the prescriptions for the treatment of acne vulgaris due to wind-heat in lung meridian from the perspective of network pharmacology. Among the commonly used drugs for the treatment of acne due to wind-heat in lung meridian, Radix scutellariae, Radix glycyrrhizae, Mori cortex, Eriobotryae folium, Fructus forsythiae, Flos lonicerae, Dried rehmannia root, Cortex moutan, Radix salviae Miltiorrhizae, and Gardeniae fructus are the most common ones, and more than half of them are due to bitter cold ${ }^{[12]}$. The meridian tropism mainly involves lung, stomach, large intestine, heart and liver meridians.

In the core prescriptions, Eriobotryae folium and Mori cortex have the effects of dispersing lung qi and clearing heat. Dried rehmannia root can clear heat and cool blood, while Gardeniae fructus can remove dampness and cool blood. Scutellariae Radix can purge fire and dry dampness. Cortex moutan can remove blood stasis and heat, activate blood and remove blood stasis. Radix Glycyrrhizae plays a mediating role. It has been proved that Loquat leaf can inhibit a variety of cocci and bacillus ${ }^{[13]}$. Paeonol and the like in cortex moutan have anti-inflammatory effect ${ }^{[14]}$. The combined use of these drugs can relieve inflammation and eliminate skin lesions. It could be seen that the core formula could treat acne from the perspective of both traditional Chinese medicine and western medicine.

The treatment of acne due to wind-heat in lung meridian should be considered from the aspects of clearing heat, purging the lung, cooling blood and detoxicating, which is consistent with the syndrome differentiation of wind-heat in lung meridian [15]. Network pharmacology research reveals that core drugs treat diseases through multi-component, multi-target and multi-channel mechanisms. This study could provide reference for the clinical application of traditional Chinese medicine and new drug research.

\section{References}

1. Purdy Sarah, Joy Langston, Lisa Tait. Presentation and management of acne in primary care: a retrospective cohort study [J]. The British journal of general practice : the journal of the Royal College of General Practitioners, 2003, 53(492).

2. J .Xia, Y.Zhu. Overview of TCM syndrome and treatment type of acne $[\mathrm{J}]$. journal of traditional chinese medicine, Hunan, 2015, 31(07): 190-192 (in Chinese).

3. M .Wang, C .Wang, W .Li, et al. Overview of acne recognition and treatment in traditional Chinese medicine [J]. Xinjiang Traditional Chinese Medicine, 2018, 36(06): 107-110 (in Chinese).

4. J. Feng, P .Zhang. Advances in the application of data mining in modern traditional chinese medicine research [J]. chinese medicine guides, 2020, 17(13): 54-57(in Chinese).

5. National Pharmacopoeia Committee. Pharmacopoeia of the People's Republic of China [M]. China Medical Science and Technology Press, (2020).

6. J. Ru, P. Li, J. Wang, et al. TCMSP: a database of systems pharmacology for drug discovery from herbal medicines [J]. Journal of Cheminformatics, 2014, 6(1).

7. Y.Xiao, M .Yang, X .Hu, et al. Compound-targetpathway network analysis and effective mechanisms prediction of Bu-Shen-Jian-Pi formula [J]. World Journal of Traditional Chinese Medicine, 2018, 4(4).

8. T.Jing, J .Liu, H .Wang, et al. Identification of blood-activating components from Xueshuan Xinmaining Tablet based on the spectrum $\tilde{A} \notin \hat{\mathrm{A}} \hat{\mathrm{A}}$ effect relationship and network pharmacology analysis [J]. RSC Advances, (2020).

9. H.Edwin, J. Anderson, J. Nathanielsz, et al. The contrasting roles of Th17 immunity in human health and disease [J]. Microbiology and immunology, 2017, 61(2).

10. H.Furusawa, S. Yoshimi, M.Yasunari, et al. Th1 and Th17 immune responses to viable Propionibacterium acnes in patients with sarcoidosis [J]. Respiratory Investigation, 2012, 50(3).

11. Y. Na, Je Sungmo, S. Seung-Hyeok. Metabolic features of macrophages in inflammatory diseases and cancer [J]. Cancer Letters,413. (2018).

12. L.Han, S.Tu, and M.Yang. Data mining based on medication regularity of acne recorded in ancient books [J]. Research of Integrated Chinese and Western Medicine, 2019, 11(05): 243-248.

13. B. Dreno, M.Richard, M. Dominique, et al. Skin microbiome and acne vulgaris : Staphylococcus , a new actor in acne [J]. Experimental Dermatology, 2017, 26(9).

14. J. Jiao, L. Sun, Z .Guo, et al. Antibacterial and anticancer PDMS surface for mammalian cell growth using the Chinese herb extract paeonol(4methoxy-2-hydroxyacetophenone) [J]. Scientific Reports, 2016, 6(1).

15. $\mathrm{T}$.Zhang, $\mathrm{Y}$.Bai. Research progress on TCM syndrome of acne vulgaris [J]. Jiangsu Traditional Chinese Medicine, 2018, 50(12): 83-86(in Chinese). 